\title{
Non-interacting main-sequence binaries with different chemical compositions: Evidences of infall of rocky material?^
}

\author{
R. G. Gratton ${ }^{1}$, G. Bonanno ${ }^{2}$, R. U. Claudi ${ }^{1}$, R. Cosentino ${ }^{2,3}$, S. Desidera ${ }^{1,4}$, \\ S. Lucatello ${ }^{1,4}$, and S. Scuderi ${ }^{2}$ \\ 1 Osservatorio Astronomico di Padova, Vicolo dell'Osservatorio 5, 35122 Padova, Italy \\ 4 Dipartimento di Astronomia, Università di Padova, Italy
}

2 Osservatorio Astrofisico, Catania, Italy

3 Centro Galileo Galilei, CNAA, Italy

Received 15 June 2001 / Accepted 16 July 2001

\begin{abstract}
We performed a careful differential abundance analysis of individual components of six main sequence binaries with separations of a few hundreds of AU. To reduce analysis concerns, we selected systems with almost equal mass components. We were able to derive differential abundances of several elements with errors down to 0.01 dex in the best cases. We found that in four systems the two components have the same chemical composition, within these very severe limits. However, clear differences were found for the two remaining systems (HD 219542 and HD 200466), in both cases the primaries being more Fe-rich than the secondaries, by $0.091 \pm 0.009$ and $0.053 \pm 0.024$ dex respectively. Similar differences were found for most of the elements considered in our analysis; however, we found no appreciable difference for volatile elements and a trend for increasing abundance differences with increasing condensation temperature for individual elements, a result similar to that found for some single stars with planets by Smith et al. (2001). Finally, we note that HD 219542A has a Li-abundance comparable to those of Li-rich stars in old open clusters, while no Li is detected in the slightly cooler HD 219542B. We suggest that the primaries of these two systems have accreted rocky planets or the inner dust-rich part of a protoplanetary disk, likely due to gravitational perturbation caused by the presence of the companion.
\end{abstract}

Key words. stars: planetary systems - stars: abundances - binaries: visual

\section{Introduction}

Accumulation of data on extrasolar planets have unvealed an unexpected correlation between presence of planets and metallicity of the central stars (see e.g. Gonzalez 1997; Butler et al. 2000). The presence of planets might be either the cause or the effect of a higher metallicity. In the first scenario (first proposed by Gonzalez 1997), the thin outer convective envelope of solar-type stars (those around

Send offprint requests to: R. G. Gratton, e-mail: gratton@pd.astro.it

* Based on observations made with the Italian Telescopio Nazionale Galileo (TNG) operated on the island of La Palma by the Centro Galileo Galilei of the CNAA (Consorzio Nazionale per l'Astronomia e l'Astrofisica) at the Spanish Observatorio del Roque de los Muchachos of the Instituto de Astrofisica de Canarias. Table 1 is only available in electronic form at the CDS via anonymous ftp to cdsarc.u-strasbg.fr $(130.79 .128 .5)$ or via http://cdsweb. u-strasbg.fr/cgi-bin/qcat?J/A+A/377/123 which planets have been discovered insofar) is enriched in metals by the ingestion of planets or part of the protoplanetary disk; this is not an exotic explanation, since orbit migration is also needed to explain the presence of giant planets close to the central stars in systems like $51 \mathrm{Peg}$ (Boss 1996; Lin et al. 1996). In the second one, the formation of planets is considered to be favoured by a higher metal-content: this might be understood in a scenario where the formation of giant planets involves first the accretion of a planetesimal and then the capture of $\mathrm{H}$ and He after a mass of 10-15 earth masses is reached (Podolak et al. 1993). Very recently, various observations have lead support to the first scenario: Israelian et al. (2001) have shown the presence of ${ }^{6} \mathrm{Li}$ in the atmosphere of the star HD 82943, around which two planets have been discovered (Naef et al. 2001): since ${ }^{6} \mathrm{Li}$ is easily destroyed in stars, Israelian et al. argue that its presence supports an external origin for this material. A further argument has been proposed by Barbieri (2001), who considered the region of formation of stars with planets (stars that are presently 
in the solar neighborhood, but possibly formed elsewhere within the Galaxy), and noticed that their Fe abundance essentially defines the upper envelope in the distribution of stellar metallicities with region of formation. Finally, Smith et al. (2001) have found that some of the stars with planets exhibit a trend for an increasing abundance excess (with respect to the Sun) with increasing condensation temperature for a given element: this trend may point to the accretion of chemically fractionated solid material into the outer (thin) convective envelope of these solartype stars. On the other side, Pinsonneault et al. (2001) found no correlation between the size of the outer convective envelope in stars with planets (expected to scale with effective temperature) and their metallicity; he argued that this is evidence against planets being the cause of the highest metallicities of stars with planets.

However, it is not easy to understand if the presence of planets causes a metal enrichment of the outer layers of single stars, because the original metal abundance is unknown, and only statistical arguments can be used. The available sample is still quite limited (even though rapidly increasing). Furthermore, any such discussion requires an homogenous analysis of a large number of systems. On the other side, wide binaries (with separation of $>50 \mathrm{AU}$, in order for protoplanetary disk to form: Nelson $2000^{1}$ ) offer a unique opportunity for a decisive test: in fact, if the presence of planets is the cause of the higher metallicity observed in the central star, we expect that in a reasonable large sample of binaries we will find some system in which the metal content of the two stars is clearly different. In order to justify the observed offset between the metallicity of stars with planets and the other stars, differences of at least $\sim 0.1$ dex are needed: the required accuracy in abundance derivations might be easily obtained in differential analysis, provided the two companions are not too much different each other. A first similar test has been presented by Laws \& Gonzalez (2001), who considered the case of $16 \mathrm{Cyg}$, whose secondary harbours a planetary system (Cochran et al. 1997). Laws \& Gonzalez found that indeed 16 Cyg B is slightly more metal-rich than 16 Cyg A (by $0.025 \pm 0.009 \mathrm{dex}$ ). However, while intriguing, this difference is small, much less than required to explain the observed offset in metallicity between stars with and without planets. It should be noticed that $16 \mathrm{Cyg} B$ has no detectable Li, while 16 Cyg A has a rather large Li content (King et al. 1997; Deliyannis et al. 2000).

A few months ago we started a systematic search for planets in binary systems with typical separations of a few hundreds of AU, using the new SARG spectrograph at the Italian TNG telescope. The motivation for this study was twofold: on one hand, we wanted to study the mechanism of formation of planets and the stability of their orbits in the presence of a perturbing companion; on the other hand, we wished to make a careful study of the difference in chemical composition

\footnotetext{
1 Marzari \& Scholl (2000) considered possible the formation of planets even in the much closer system of $\alpha$ Cen.
}

between the two components. For this reason, we selected a sample of solar-type binaries with accurate parallaxes taken from the Hipparcos Multiple Star Catalogue (available at CDS). Selection criteria considered systems with separation larger than 2 arcsec (to avoid contamination of the spectra) and magnitude difference less than 1.0 mag: since we limited ourselves to unevolved stars, this ensures that the temperature differences between the two stars do not exceed $\sim 350 \mathrm{~K}$ : these stars are then very well suited for a careful differential abundance analysis. We further limited ourselves to stars whose components have colours in the range $0.45<(B-V)<1.1$ (again, limiting to solar type stars we improve reliability of the abundance analysis), parallaxes larger than 10 mas (with errors smaller than 5 mas), and declination $\delta>-20$. Typical projected separations are in the range 100-1000 AU. Dynamical stability for a planet up to 20-30 AU from the star is possible in most cases, according to the simulations of Holman \& Wiegert (1999). Among the planets already discovered, one orbits in a binary system with projected separation of 150 AU (HD 195019: Fischer et al. 1999). Our original list included about 60 systems: the final sample of the survey is selected using SARG observations. We are checking for undetected double line binaries, large radial velocity differences between the two components, rotational broadening and activity indicators. While we indeed rejected from the whole sample a few stars based on these criteria, none of the stars in the six systems we will discuss in this paper is a double line binary, and the difference in radial velocities between the two components $\left(<1.5 \mathrm{~km} \mathrm{~s}^{-1}\right)$ is compatible with orbital motion due to the previously known companion. Note however that the difference in radial velocities between the two components of HD 13357 does show a trend to increase with time: we suspect that one of the components (most likely B) is itself a spectroscopic binary. Rotation is undetected at a very low level in most systems; however, lines in the spectra of the two components of HD 8071 are likely rotationally broadened (we determined values of $v \sin i=8$ and $7 \mathrm{~km} \mathrm{~s}^{-1}$ for components $\mathrm{A}$ and $\mathrm{B}$ respectively).

\section{Observations}

The spectra used in the present analysis are the template spectra needed for the high precision radial velocity measurements to be used for planet search. Observations were obtained in October and November 2000 using the SARG high resolution spectrograph (Gratton et al. 2001) at Telescopio Nazionale Galileo, in La Palma. The spectra were obtained using the Yellow Grism, and cover the wavelength range $4700-7900 \AA$ at a resolving power of $\sim 160000$ (this is the value obtained from the instrumental profile modelling by the AUSTRAL code used for high precision radial velocities: Endl et al. 2000). Exposure times were limited to $1 \mathrm{hr}$, to avoid smearing of lines due to the Earth rotation, save for HD 8071 (30 min for both components), HD 33334A (50 min), and HD 200466 (15 min for both components). Peak $S / N$ of the spectra are in the 
Table 1. Equivalent widths for program stars. (Table 1 is only available in electronic form at the CDS via anonymous ftp to cdsarc.u-strasbg.fr (130.79.128.5) or via http://cdsweb.u-strasbg.fr/cgi-bin/qcat?J/A+A/377/123).

range 100-300 (see Table 2). To reduce possible light contamination between the two components, the slit was kept roughly perpendicular to the line joining the images of the two components of each system; even so, there might be some contamination (at $<1 \%$ anyhow) between the two components of HD 8071 (the separation is 2.2 arcsec, and the spectra were obtained with a seeing of 1.5 arcsec $F W H M$ ); in all other cases, separation is $>4.5$ arcsec, so that there was no concern.

Individual orders were extracted using standard routines for reduction of echelle spectra within $\mathrm{IRAF}^{2}$. The unidimensional spectra were then analyzed using routines within the ISA code (Gratton 1988): continuum was obtained by fitting a cubic spline through automatically selected regions in the spectra (however, discrepant points as given by a visual inspection were removed from the fit). Lines were then measured using an automatic procedure, which estimates a local continuum level and fits the the lines with a Gaussian. Only lines with equivalent widths $E W<50 \mathrm{~m} \AA$ were used in the final analysis, so that there is no concern about damping wings. $E W$ s used in the final analysis are listed in Table 1, available in electronic form at CDS.

\section{Analysis}

\subsection{Basic parameters}

Table 2 lists the most interesting parameters for the program stars. Columns 2 and 3 are the absolute magnitudes $M_{V}$, obtained from the absolute magnitudes and parallaxes listed in the Hipparcos catalogue. Columns 4 and 5 are the masses, calculated from $M_{V}$ magnitudes and $B-V$ colours using Gray (1992) tables. Columns 6 and 7 are the $B-V$ colours from the Hipparcos catalogue. Columns 8 and 9 are the effective temperatures used in our analysis: they were derived from the Fe equilibrium of ionization (see discussion below). Columns 10 and 11 are the surface gravities: they were derived from the definition of effective temperatures, and from the basic relation between masses, radii and gravities. The bolometric corrections BC were from Kurucz (1995). Columns 12 and 13 give the overall metal abundance used in the abundance analysis. Finally Cols. 14 and 15 give the peak $S / N$ of our spectra.

\subsection{Abundance analysis}

Our analysis is differential with respect to the Sun, and uses Kurucz (1995) model atmospheres, computed with the overshooting option switched off for both the Sun and the program stars (Castelli et al. 1997). In the following

${ }^{2}$ IRAF is distributed by the NOAO, which are operated by AURA, under contract with NSF.

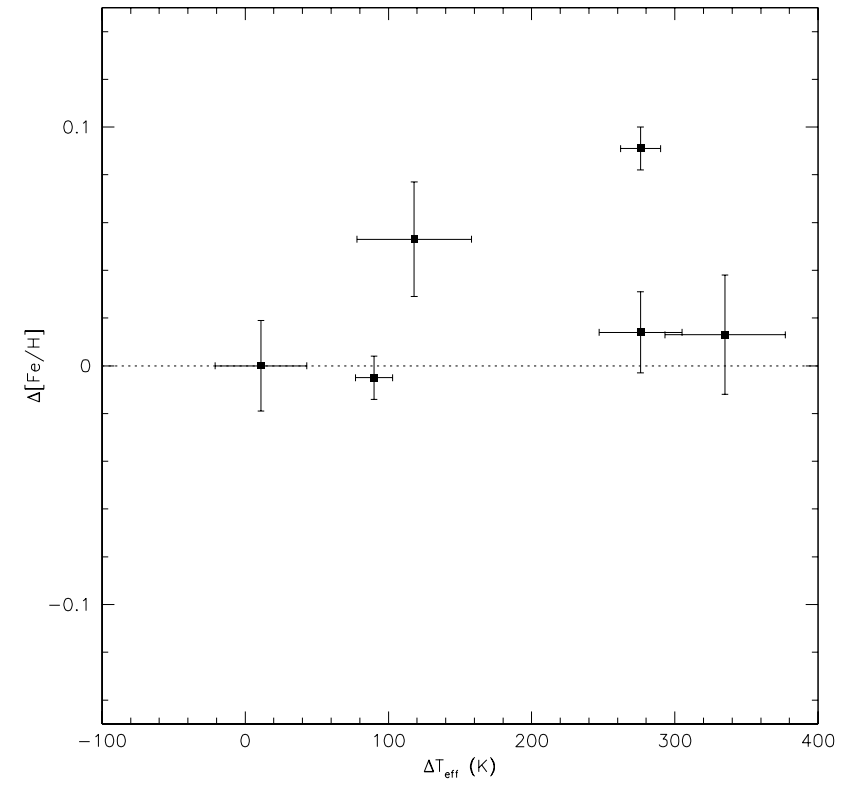

Fig. 1. Run of the differences between the abundances obtained for the two components of each system with the difference in the effective temperatures adopted for the stars.

discussion we are essentially interested in the comparison between the companions in each system, and hence we invite the reader to concentrate on this issue.

The most important ingredient in our differential analysis is the difference in temperature between the two components of each binary. For our stars, we may take advantage of the knowledge of the luminosity difference between the two components, since they are at the same distance from us. This allows a very sensitive differential analysis between the two components, improving over a standard spectroscopic analysis, like that of e.g. Laws \& Gonzalez (2001) for 16 Cyg. In fact, since the stars are on the main sequence (and then we know the ratio between the masses of the two stars with small errors), we may then obtain accurate estimates of the ratio between the surface gravities in the two systems (provided we have a good guess on the effective temperature of the stars). This opens the possibility to accurately derive the difference of the temperature of the two stars using the equilibrium of ionization (e.g. for $\mathrm{Fe}$ ) because the difference between abundances provided by neutral and singly ionized Fe lines has a strong temperature sensitivity $(\sim 0.0009 \mathrm{dex} / \mathrm{K}$ for the line lists used throughout this paper $)^{3}$. An iterative procedure is required (because gravities depend on the assumed temperatures), but the process converges rapidly. The accuracy of these temperature differences is mainly limited by three issues: (i) by the line-to-line scatter in abundances;

\footnotetext{
3 In the analysis of the primaries, we adopted the same procedure comparing the star to the Sun; of course, some of the advantages of our strictly differential approach are lost: for this reason, the absolute abundances we get for the systems have much larger associated errors than the differential abundances between individual components.
} 
Table 2. Basic stellar parameters.

\begin{tabular}{|c|c|c|c|c|c|c|c|c|c|c|c|c|c|c|}
\hline Star & $\begin{array}{c}M_{V} \\
\mathrm{~A}\end{array}$ & $\begin{array}{c}M_{V} \\
\mathrm{~B}\end{array}$ & $\begin{array}{c}\text { Mass } \\
\text { A }\end{array}$ & $\begin{array}{c}\text { Mass } \\
\text { B }\end{array}$ & $\begin{array}{c}B-V \\
\mathrm{~A}\end{array}$ & $\begin{array}{c}B-V \\
\mathrm{~B}\end{array}$ & $\begin{array}{c}T_{\text {eff }} \\
\text { A }\end{array}$ & $\begin{array}{c}T_{\text {eff }} \\
\text { B }\end{array}$ & $\begin{array}{c}\log g \\
\mathrm{~A}\end{array}$ & $\begin{array}{c}\log g \\
\text { B }\end{array}$ & $\begin{array}{c}{[\mathrm{A} / \mathrm{H}]} \\
\mathrm{A}\end{array}$ & $\begin{array}{c}{[\mathrm{A} / \mathrm{H}]} \\
\mathrm{B}\end{array}$ & $\begin{array}{c}S / N \\
\mathrm{~A}\end{array}$ & $\begin{array}{c}S / N \\
\mathrm{~B}\end{array}$ \\
\hline HD 008071 & 3.82 & 4.05 & 1.15 & 1.10 & 0.631 & 0.654 & 6103 & 6092 & 4.23 & 4.30 & 0.26 & 0.26 & 236 & 161 \\
\hline HD 013357 & 4.80 & 5.27 & 0.97 & 0.89 & 0.747 & 0.817 & 5653 & 5377 & 4.36 & 4.41 & 0.03 & 0.03 & 105 & 113 \\
\hline HD 030101 & 5.53 & 5.68 & 0.81 & 0.80 & 0.928 & 1.039 & 5080 & 4990 & 4.30 & 4.26 & -0.17 & -0.17 & 133 & 192 \\
\hline HD 033334 & 4.73 & 5.55 & 0.95 & 0.84 & 0.783 & 0.900 & 5638 & 5303 & 4.27 & 4.42 & 0.09 & 0.09 & 292 & 230 \\
\hline HD 200466 & 5.21 & 5.36 & 0.91 & 0.86 & 0.787 & 0.883 & 5533 & 5415 & 4.45 & 4.38 & 0.05 & 0.00 & 101 & 94 \\
\hline HD 219542 & 4.48 & 4.87 & 1.02 & 0.93 & 0.705 & 0.791 & 5989 & 5713 & 4.37 & 4.38 & 0.28 & 0.18 & 235 & 229 \\
\hline
\end{tabular}

(ii) by the accuracy of the luminosity ratio between the two components; and (iii) by systematic changes with temperatures of the assumption on which equilibrium of ionization calculations are based (e.g. deviations of real atmospheres from model ones, or departures from LTE).

To reduce the first concern, our analysis is strictly differential, that is we considered exactly the same set of lines in both components of a binary and only considered the difference between the abundances obtained from the two components, line-by-line. We used the same objective criteria to trace the continuum in the two stars, and used an automatic routine for measuring equivalent widths, with all parameters set equal when considering the two stars. Also, we limited our analysis to lines with $E W<50 \mathrm{m \AA}$ (to avoid lines that are too saturated, and are then more sensitive to velocity fields than to abundances; or have well-developed damping wings, not well fitted by our Gaussian fitting routine). We took full advantage of the wide spectral range and high resolution offered by SARG, so that in spite of these criteria, our line lists include several hundreds of lines for each star, ensuring very small statistical error bars.

Note that errors in parallaxes affect gravities derived from both components in the same way; hence there is no effect on the derived temperature differences. On the other hand, when comparing the stars to the Sun, errors in parallaxes have to be taken into account: for this reason errors in the temperatures with respect to the Sun are $40-50 \mathrm{~K}$ for all systems (this is larger than the error in the difference between the temperature of the two components).

As to the third issue, we admit that systematic trends might be expected, with increasing differences of the derived abundances with increasing temperature differences between the two components. However, we think that the impact of such trends is small if any. To show this, first we compared temperature differences obtained from the equilibrium of ionization with values obtained using independent techniques: equilibrium of excitation (for Fe I), $B-V$ and $V-I$ colours (from the Hipparcos catalogue and Cuypers \& Seggewiss 1999, respectively), and from the difference in magnitude between the two components times the slope of the main sequence (see Table 3 ). While each of these methods has its pro's and con's, on the whole the agreement between these various sets of temperatures
Table 3. Temperature differences between components obtained using various methods: Second column: ionization equilibrium for Fe; Third column: excitation equilibrium for Fe I; Fourth column: temperatures from Hipparcos $B-V$ colours, using the temperature calibration by Kurucz (1995); Fifth column: temperatures obtained from the difference in magnitude between the components, times the slope of the zero age main sequence; Sixth column: temperatures from $V-I$ colours (Cuypers \& Seggewiss 1999) using the calibration by Alonso et al. (1996) (Cousins $V-I$ colours were transformed into Johnson $V-I$ colours using the calibration by Bessel 1983). Note that Hipparcos $B-V$ colours are not very accurate because our systems are not resolved by the Hipparcos photometric beam; and temperatures from the difference in magnitude between the two components assume that they are unevolved, and are then upper limits to the actual temperature differences.

\begin{tabular}{lccccc}
\hline Star & Ion. eq. & Exc. eq. & $B-V$ & $\Delta V$ & $V-I$ \\
\hline HD 008071 & $11 \pm 32$ & $106 \pm 108$ & 90 & 180 & \\
HD 013357 & $276 \pm 29$ & $159 \pm 59$ & 209 & 281 & \\
HD 030101 & $90 \pm 13$ & $117 \pm 27$ & 306 & 83 & 121 \\
HD 033334 & $335 \pm 42$ & $354 \pm 84$ & 350 & 448 & \\
HD 200466 & $118 \pm 40$ & $102 \pm 117$ & 340 & 106 & \\
HD 219542 & $276 \pm 14$ & $280 \pm 37$ & 298 & 270 & 238 \\
\hline
\end{tabular}

is good, supporting our adopted temperature differences. Second, we examined the run of the difference of the abundances between the two components (in the sense primarysecondary) with the difference in temperature obtained for the two stars (see Fig. 1). In four systems there is a very good agreement between the abundances obtained from the two components: they span the whole range of temperature differences between the two components. A full discussion of any systematic trend should clearly require a larger sample: for the time being, we think that such systematic difference should not exceed 0.02 dex for a temperature difference as large as $350 \mathrm{~K}$, and it is possibly less.

Finally, the same value of the microturbulent velocity was adopted for all stars.

\subsection{Iron}

Table 4 lists the average $\mathrm{Fe}$ abundances obtained for each component of the six systems, along with the rms 
Table 4. Derived $[\mathrm{Fe} / \mathrm{H}]$ values.

\begin{tabular}{lcccccc}
\hline Star & A & rms & B & rms & A-B & rms \\
\hline HD 008071 & $+0.258 \pm 0.016$ & 0.130 & $+0.258 \pm 0.017$ & 0.137 & $+0.000 \pm 0.010$ & 0.082 \\
HD 013357 & $+0.031 \pm 0.013$ & 0.111 & $+0.018 \pm 0.011$ & 0.095 & $+0.014 \pm 0.009$ & 0.073 \\
HD 030101 & $-0.172 \pm 0.011$ & 0.091 & $-0.167 \pm 0.011$ & 0.095 & $-0.005 \pm 0.005$ & 0.040 \\
HD 033334 & $+0.095 \pm 0.012$ & 0.095 & $+0.082 \pm 0.011$ & 0.086 & $+0.013 \pm 0.013$ & 0.107 \\
HD 200466 & $+0.048 \pm 0.012$ & 0.083 & $-0.005 \pm 0.014$ & 0.097 & $+0.053 \pm 0.014$ & 0.097 \\
HD 219542 & $+0.293 \pm 0.014$ & 0.121 & $+0.201 \pm 0.013$ & 0.111 & $+0.091 \pm 0.006$ & 0.048 \\
\hline
\end{tabular}

Table 5. Best Fe abundance differences within each system.

\begin{tabular}{lc}
\hline Star & {$[\mathrm{Fe} / \mathrm{H}] \mathrm{A}-[\mathrm{Fe} / \mathrm{H}] \mathrm{B}$} \\
\hline HD 008071 & $+0.000 \pm 0.019$ \\
HD 013357 & $+0.014 \pm 0.017$ \\
HD 030101 & $-0.005 \pm 0.009$ \\
HD 033334 & $+0.013 \pm 0.025$ \\
HD 200466 & $+0.053 \pm 0.024$ \\
HD 219542 & $+0.091 \pm 0.009$ \\
\hline
\end{tabular}

scatter of individual values around the mean (typically about 60 Fe I lines were used for each system). We note that while the scatter for individual lines in the abundance analysis of each component are those typically encountered in abundance analysis of solar-type stars with good $S / N$ spectra $(\sim 0.1 \mathrm{dex})$, the rms scatter for the difference is generally lower, and in the best cases is as low as 0.04 dex. This is because we are performing a strictly differential analysis, so that several sources of errors cancel out.

The error bars given in Col. 6 of Table 4 only take into account the line-to-line scatter. However, the real error bars should also consider the effects of errors in the adopted atmospheric parameters (mainly due to errors in the effective temperatures). This was done by multiplying the expected errors in the temperature difference between the two stars for the sensitivity of abundances on temperatures, and summing quadratically this term to that due to the line-to-line scatter. The resulting error bars are listed in Table 5. Inspection of this table shows that while we found no difference between the iron content of the two components for four systems, we obtain rather large differences for the remaining two.

The case of HD 219542, with a difference significative at more than $10 \sigma$, is of particular interest, because the difference is of the same order of magnitude needed to explain the systematic offset between the abundances of stars with and without planets. The difference is obviously much larger than given by line-to-line scatter (see Fig. 2). Of course, this large difference is based on the assumed temperature difference of $276 \pm 14 \mathrm{~K}$ between the two components. In order to cancel out the difference we found, the temperature difference between the two components
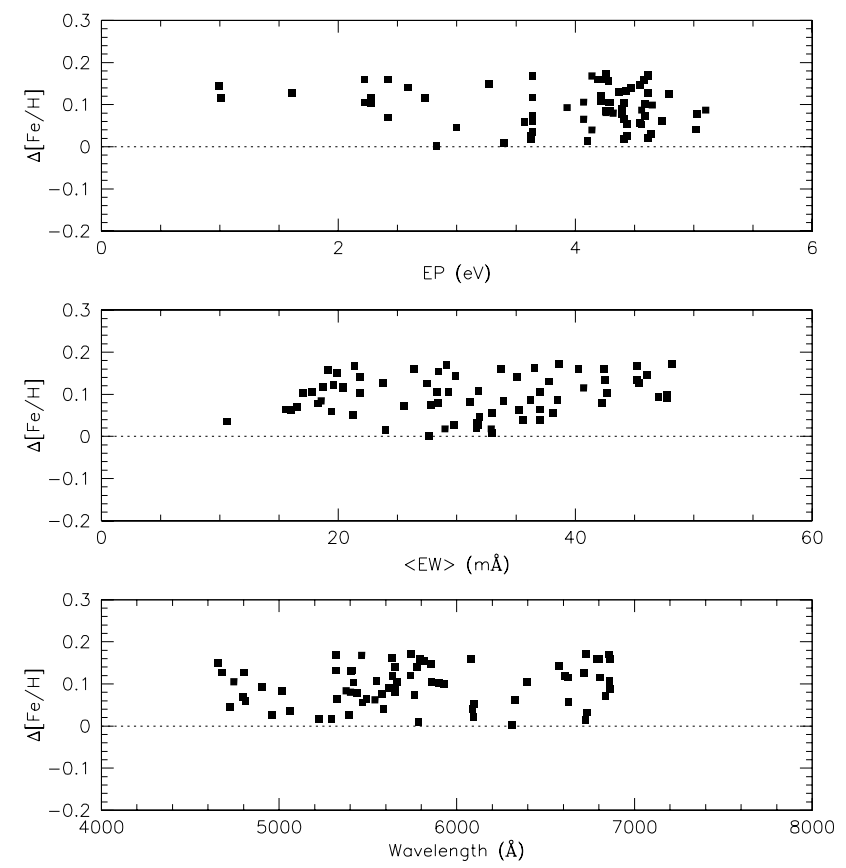

Fig. 2. Difference between the Iron abundance (from Fe I lines) in the two components of HD $219542 \Delta[\mathrm{Fe} / \mathrm{H}]$ as a function of excitation potential, equivalent width (average in the two components), and wavelength. There is a clear offset from zero in all these diagrams, and no obvious trend.

of this system should be reduced to a value $\leq 100 \mathrm{~K}$. This seems unlikely for a number of reasons:

- For this star, there is an excellent agreement among all temperature indicators for a temperature difference close to the adopted one (the average of the other four indicators listed in Table 3 is $272 \mathrm{~K}$, very close to the value of $276 \pm 14$ given by the equilibrium of ionization);

- Figure 3 shows the location of the two components of HD 219542 on the $T_{\text {eff }}-M_{V}$ diagram. For each component we give two error bars: the thin line gives the error bar in the absolute location of the stars in this diagram; the thick error bar is related to errors in the relative position between the two stars (assuming an error of $0.05 \mathrm{mag}$ in the relative photometry). The position of the two stars agree very well with a 1 Gyr old isochrone for $[\mathrm{Fe} / \mathrm{H}]=+0.2$ (from Bertelli et al. 2000): there is no room for the primary actually 


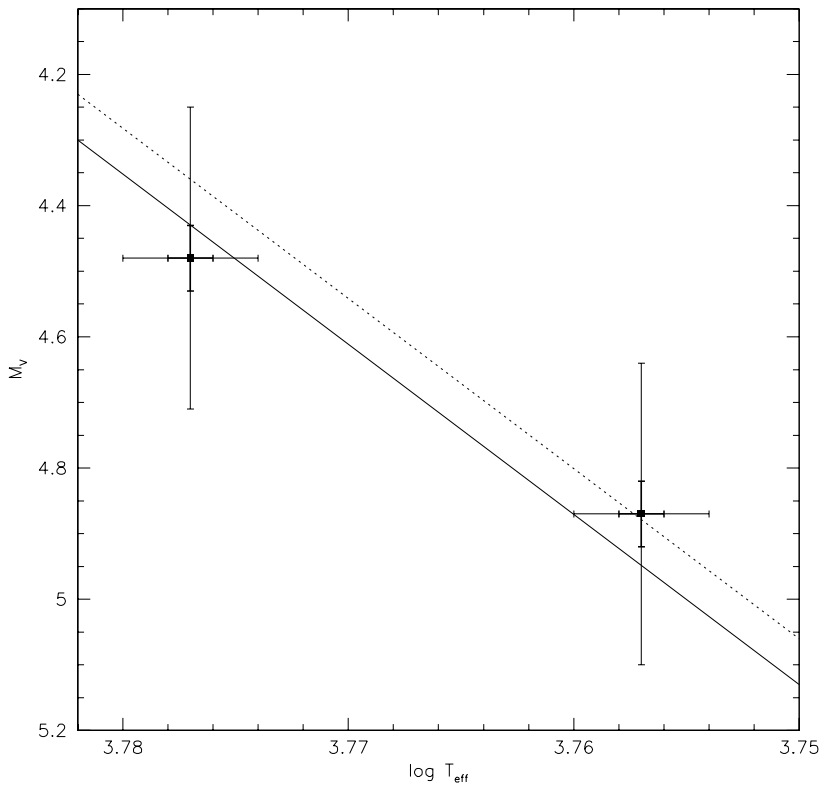

Fig. 3. Location of the two components of HD 219542 on the $T_{\text {eff }}-M_{V}$ diagram. For each component we give two error bars: the longer thin line is the error bar in the absolute location of the stars in this diagram; the shorter thick error bar is related to errors in the relative position between the two stars (assuming an error of $0.05 \mathrm{mag}$ in the relative photometry). Solid and dashed lines represent the 1 Gyr old isochrone for $[\mathrm{Fe} / \mathrm{H}]=+0.2$ and $[\mathrm{Fe} / \mathrm{H}]=+0.3$ respectively (from Bertelli et al. 2000).

being an equal mass binary (as it is needed in order to cancel out the metal abundance difference found in our analysis). Also, the location of the two stars is well fitted by isochrones that assume the same chemical composition for the two components: for comparison, the dashed line give the location of the 1 Gyr old isochrone with $[\mathrm{Fe} / \mathrm{H}]=+0.3$, that is clearly too red to fit HD 219542A;

- a high level of activity might introduce systematic errors in abundance analysis at $\sim 0.1$ dex level (Cayrel et al. 1985). Wilson (1963) assigned an activity indicator value of $I(H K)=0$ to both components of HD 219542, a value indicating absence of chromospheric activity. We have further indication that the activity is low in these stars. Activity is known to decay with age; the age of HD 219542 cannot be determined accurately, since both components are still very close to the Zero Age Main Sequence in the $T_{\text {eff }}-M_{V}$ diagram. However, line profiles do not show any sign of rotational broadening. A very generous upper limit is obtained neglecting the broadening contribution due to macroturbulence (that rather likely is the dominant cause of line broadening in both stars). In this way, we get upper limits of 3.7 and $3.4 \mathrm{~km} \mathrm{~s}^{-1}$ for $v \sin i$ for components A and B respectively. These values are much smaller than typical rotation in Hyades stars of the same colour (Soderblom et al. 1993). This argues against a very young age for this system

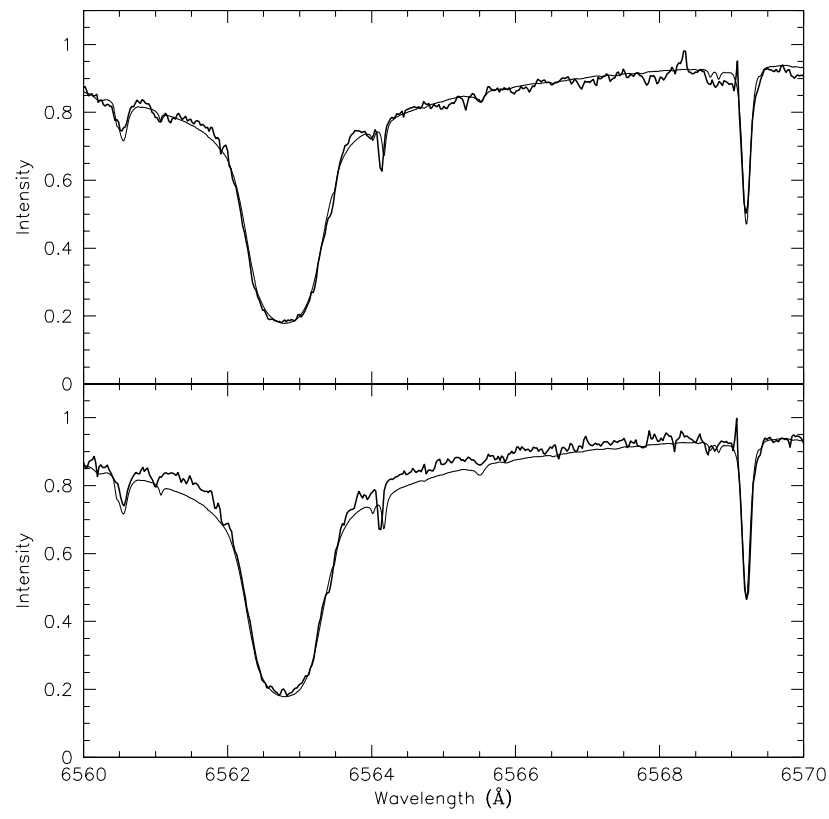

Fig. 4. Observed spectra in the region of $\mathrm{H}_{\alpha}$ for the two components of HD 219542 (a) upper panel; b) lower panel: thick lines), compared with the solar flux spectrum (Kurucz et al. 1984: thin lines).

(an age of $\sim 2$ Gyr is obtained from the Skumanich law). Furthermore, there is no sign of anomalous emission in the core of $\mathrm{H}_{\alpha}$ (see Fig. 4, where it is shown that both components of HD 219542 display $\mathrm{H}_{\alpha}$ cores very similar to that obtained for the Solar flux spectrum). We conclude that it is not probable that either of the two components of HD 219542 is an active star and that this is the cause of the offset in abundance we found between the two stars. However, we have started a photometric monitoring of this system looking for activity-related variability

We conclude that the large difference between the metal abundance of the two components is very likely real.

\subsection{Other elements}

When considering abundances of various elements, we divided elements into four groups (refractary, siderophiles, silicates, and volatiles), according to the scheme usually adopted in the analysis of the chemical composition of meteorites (see e.g. Anders \& Grevesse 1989: Table 6). For each star, we averaged abundance differences weighting each element according to the number of measured lines. Finally, we also calculated the average for the four binaries for which we did not find any difference between the iron abundance of the two components, weighting them according to the number of lines and the rms of abundance differences from individual lines, obtained for Fe.

From Table 6 we obtain the following results:

- For "normal" binaries, that is those in which there is no significative difference in Fe abundance between 
Table 6. Average abundance differences $(\mathrm{A}-\mathrm{B})$ for different groups of elements.

\begin{tabular}{|c|c|c|c|c|c|c|c|c|}
\hline Star & & refractary & & siderophiles & & silicates & & volatiles \\
\hline HD 008071 & 44 & $-0.016 \pm 0.012$ & 126 & $-0.012 \pm 0.007$ & 3 & $0.082 \pm 0.047$ & 9 & $-0.019 \pm 0.027$ \\
\hline HD 013357 & 61 & $-0.013 \pm 0.010$ & 163 & $0.009 \pm 0.006$ & 13 & $-0.046 \pm 0.022$ & 9 & $-0.022 \pm 0.026$ \\
\hline HD 030101 & 40 & $-0.007 \pm 0.006$ & 151 & $-0.005 \pm 0.003$ & 11 & $-0.015 \pm 0.012$ & 6 & $0.050 \pm 0.016$ \\
\hline HD 033334 & 47 & $-0.042 \pm 0.016$ & 135 & $-0.005 \pm 0.009$ & 9 & $0.034 \pm 0.036$ & 9 & $-0.133 \pm 0.036$ \\
\hline normal & 192 & $-0.013 \pm 0.005$ & 575 & $-0.003 \pm 0.003$ & 36 & $-0.013 \pm 0.010$ & 33 & $0.004 \pm 0.012$ \\
\hline HD 219542 & 55 & $0.055 \pm 0.006$ & 155 & $0.080 \pm 0.004$ & 6 & $0.050 \pm 0.020$ & 9 & $0.008 \pm 0.016$ \\
\hline HD 200466 & 50 & $0.077 \pm 0.014$ & 111 & $0.071 \pm 0.009$ & 7 & $0.044 \pm 0.037$ & 4 & $-0.067 \pm 0.049$ \\
\hline
\end{tabular}

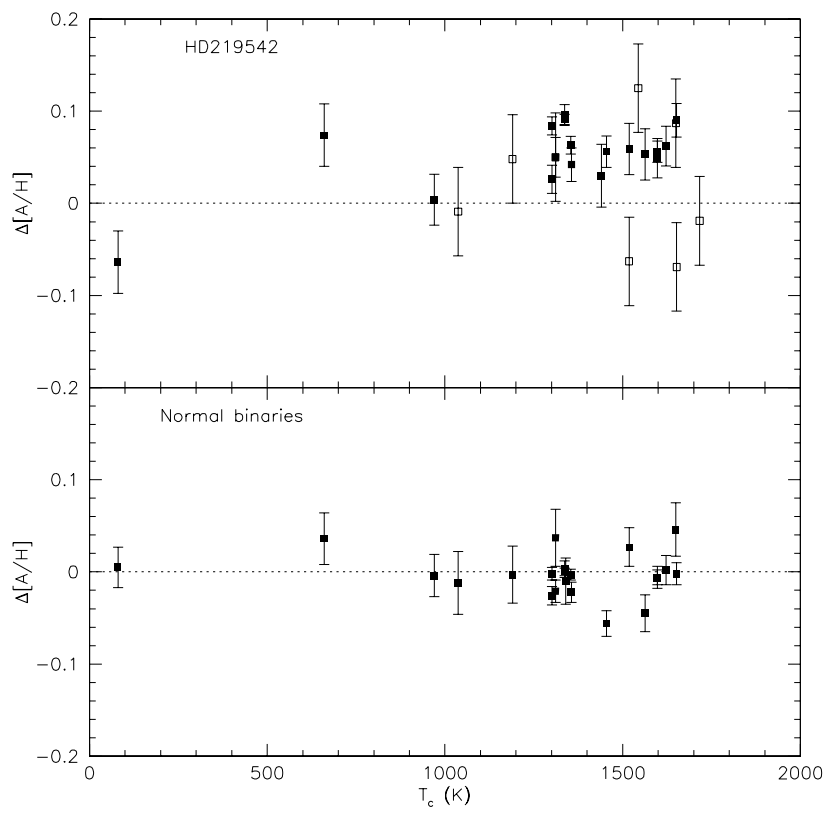

Fig. 5. Run of the difference in abundances between the two components $(\mathrm{A}-\mathrm{B})$ as a function of the condensation temperature $T_{\mathrm{c}}$ for the elements considered in the present analysis for HD 219542 (upper panel) and "normal" binaries, whose components have the same iron abundance (lower panel). Filled symbols are elements for which more than one line was measured; open symbols are elements for which only one line was considered.

the two components, differences in abundances for the other elements are also small, likely not significative;

- for those two binaries where significant differences of $\mathrm{Fe}$ abundances between the two components exist, there are significant differences in siderophiles, refractary, and silicate elements, while there is apparently not a significant difference in the abundances of volatile elements (here, $\mathrm{C}, \mathrm{Na}, \mathrm{Mn}, \mathrm{Cu}$, and $\mathrm{Zn}$ ). This result is most clear for HD 219542, the star for which we have better observational material. Another way to display this result is given in Fig. 5, where we plotted the run of the difference in abundances between the two components $(\mathrm{A}-\mathrm{B})$ as a function of the condensation temperature $T_{\mathrm{c}}$ for the elements considered
Table 7. Lithium abundances.

\begin{tabular}{lcc}
\hline Star & A & B \\
\hline HD 008071 & 2.75 & 2.80 \\
HD 013357 & 1.75 & 0.70 \\
HD 030101 & $<0.5$ & $<0.5$ \\
HD 033334 & 1.15 & $<1.0$ \\
HD 200466 & & \\
HD 219542 & 2.35 & $<1.0$ \\
\hline
\end{tabular}

in the present analysis. There is a defined trend for a larger abundance difference with increasing condensation temperature; however a weighted least squares fit shows that the significance of this trend is quite marginal (a bit more than $1 \sigma$ ).

\subsection{Lithium abundances}

Lithium abundances were obtained by comparing the observed spectra in the region of the Li resonance doublet with appropriate synthetic spectra. An example of these comparisons is shown in Fig. 6. Results are listed in Table 7. The case of HD 219542 (the binary with the largest difference in $\mathrm{Fe}$ abundance between the two components) is of particular interest. If we compare the $\mathrm{Li}$ abundances for the two components of this system with those of the old open cluster M 67 (see Fig. 7), we find that while the Fe-richer component $\mathrm{A}$ has a $\mathrm{Li}$ abundance comparable to those of Li-rich stars in $\mathrm{M} 67^{4}$, no $\mathrm{Li}$ is detected in the Fe-poorer component. This will place this star amongst the Li-poor stars in M 67.

\section{Discussion and conclusions}

We examined the chemical composition of the components of six wide binaries, using a very precise differential analysis, able to unveal small abundance differences between the two components. In four out of six systems, the composition of the two components is the same within narrow error bars. In the remaining two systems (HD 219542

\footnotetext{
4 Unluckily, $S / N$ of our spectra is too low for an appropriate analysis of the ${ }^{6} \mathrm{Li}$ content.
} 


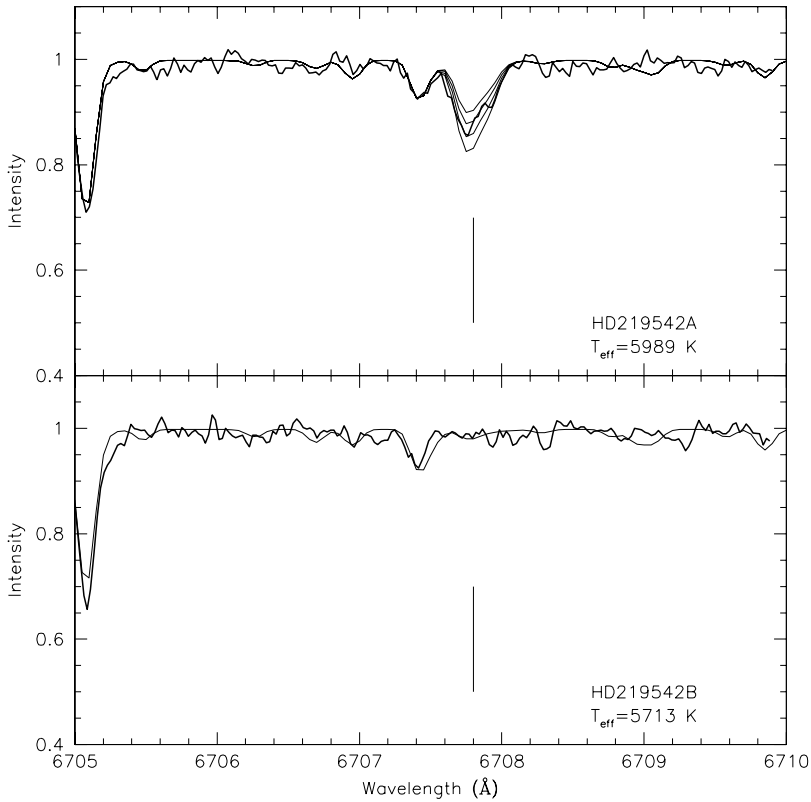

Fig. 6. Comparison between the observed spectra of HD 219542A and B (thick lines in the upper and lower panel respectively) and synthetic spectra (thin lines) in the region of the $\mathrm{Li}$ resonance doublet, whose location is marked by a vertical line. Synthetic spectra were computed with atmospheric parameters appropriate for the stars, and $\mathrm{Li}$ abundances of $\log n(\mathrm{Li})=2.2,2.3,2.4$, and 2.5 in the upper panel; and $\log n(\mathrm{Li})=1.0$ in the lower panel (these $\mathrm{Li}$ abundances are in the usual scale where $\log n(\mathrm{H})=12$ ).

and HD 200466) the primary is more iron-rich than the secondary. The case of HD 219542 is very strong, the difference being significant at about $10 \sigma$. Such a difference cannot be due to errors in the analysis, or to an insofar undetected stellar companion around one of the two stars. When considering all the elements for which we have data, we found that while siderophiles, refractary, and silicate elements are clearly overabundant in the primaries of these two systems, there is apparently not a significant difference in the abundances of volatile elements (again, the case of HD 219542 is most clear). We also note that the two components of HD 219542 have a very different $\mathrm{Li}$ abundance: while HD 219542A has a Li abundance similar to that measured in Li-rich stars in old open clusters, no $\mathrm{Li}$ is detected in the secondary of this system, in analogy with Li-poor systems in old open clusters.

It appears that HD 219542 is similar to the group of stars with planets studied by Smith et al. (2001). Our results suggest that the difference in the chemical composition between the two components of HD 219542 and possibly HD 200466 is due to ingestion by the primaries of dust-rich or rocky material, coming from either the inner part of a proto-planetary disk or from rocky planets. In the case of HD 219542A, that has a thin convective envelope (see e.g. Pinsonneault et al. 2001), we estimate that ingestion of a few $(\sim 3-4)$ earth masses of rocky material are enough to justify the observed abundance pattern

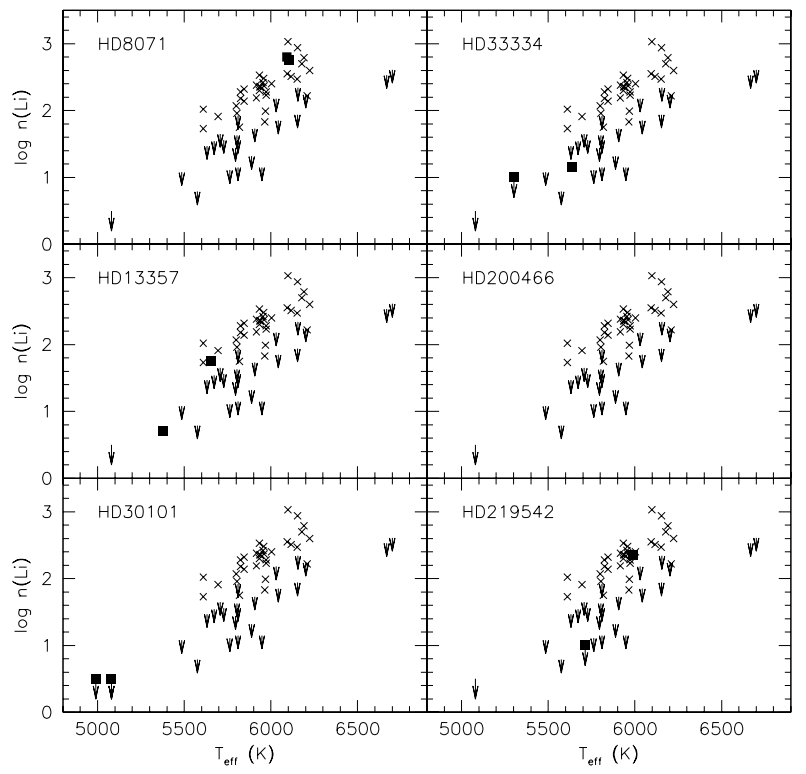

Fig. 7. Run of the Li abundances with effective temperature for the program stars (filled squares), compared with $\mathrm{Li}$ abundances in stars in the old open cluster M 67 (crosses: from Jones et al. 1999). Arrows represent upper limits.

if this ingestion occurred after convection receded to the thin outer layers of the star. Addition of external material would help producing a larger Li content in this star. On the other hand, the Li-planets connection is still far from being well understood (see the discussion in Laws \& Gonzalez 2001 for the case of 16 Cyg). Searching for planets around stars in these systems (as well as in the others here considered) is presently actively performed at SARG.

Acknowledgements. We thank Eugenio Carretta for a critical reading of the paper. This research has made use of the SIMBAD data base, operated at CDS, Starsbourg, France. Partial support by CNAA is also acknowledged.

\section{References}

Alonso, A., Arribas, S., \& Martinez-Roger, C. 1996, A\&A, 313, 873

Anders, \& Grevesse 1989, Geochem. Cosmochem. Acta, 53, 197

Barbieri, M. 2001, private communication

Bertelli, P., Girardi, L., Bressan, A., Chiosi, C., Nasi, E. 2000, A\&AS, 141, 371

Bessell, M. S. 1983, PASP, 95, 480

Boss, A. P. 1996, Lunar Planet. Sci., 27, 139

Butler, R. P., Vogt, S., Marcy, G. W., et al. 2000, ApJ, 545, 504

Castelli, F., Gratton, R. G., \& Kurucz, R. L. 1997, A\&A, 318, 841

Cayrel, R., Cayrel de Strobel, G., \& Campbell, R. 1985, A\&A, 146,249

Cochran, W. D., Hatzes, A. P., Butler, R. P., \& Marcy, G. W. 1997, ApJ, 483, 457

Cuypers, J., \& Seggewiss, W. 1999, A\&AS, 139, 425

Deliyannis, C. P., Cunha, K., King, J. R., \& Boesgaard, A. M. 2000, AJ, 119, 2437 
Endl, M., Kürtser, M., \& Els, S. 2000, A\&A, 362, 585

Fischer, D. A., Marcy, G. W., Butler, R. P., Vogt, S., \& Apps, K. 1999, PASP, 111, 50

Gonzalez, G. 1997, MNRAS, 285, 403

Gratton, R. G. 1988, Rome Obs. Preprint, 29

Gratton, et al. 2001, in preparation

Gray, D. F. 1992, The Observation and Analysis of Stellar Photospheres (Cambridge University Press, Cambridge)

Holman, P. A., \& Wiegert, M. J. 1999, AJ, 117, 621

Israelian, G, Santos, N. C., Mayor, M., \& Rebolo, R. 2001, Nature, 411, 163

King, J. R., Deliyannis, C. P., Hiltgen, D. D., et al. 1997, AJ, 113,1871

Kurucz, R. L. 1995, CD-ROM 13

Kurucz, R. L., Furenlid, I., \& Brault, J. 1984, Solar Flux Atlas from 296 to 1300 nm, National Solar Observatory, Sunspot, $\mathrm{NM}$
Laws, C., \& Gonzalez, G. 2001, ApJ, 553, 405

Lin, D. N. C., Bodenheimer, P., \& Richardson, D. C. 1996, Nature, 380, 606

Marzari, F., \& Scholl, H. 2000, ApJ, 543, 328

Naef, D., et al. 2001, A\&A, in press

Nelson, A. F. 2000, ApJ, 537, L65

Pinsonneault, M. H., DePoy, D. L., \& Coffee, M. 2001, ApJL, in press [astro-ph/0105439]

Podolak, M., Hubbard, W. B., \& Pollack, J. B. 1993, in Protostars and Planets III, ed. E. H. Levy, \& J. L. Lunine (Univ. Arizona Press, Tucson), 1109

Soderblom, D. R., Stauffer, J. R., McGregor, K. B., \& Jones, B. F. 1993, ApJ, 409, 624

Smith, V. V., Cunha, K., \& Lazzaro, D. 2001, AJ, in press [astro-ph/0103063]

Wilson, O. C. 1963, ApJ, 138, 832 